\title{
New spectroscopic components in 8 multiple systems ${ }^{\star}$
}

\author{
A.A. Tokovinin ${ }^{1,2}$ \\ 1 Sternberg Astronomical Institute, 13 Universitetsky prosp., 119899 Moscow, Russia \\ e-mail: toko@sai.msu.su \\ 2 Centre de Recherche Astronomique de Lyon, UMR CNRS 5574, 9 Av. Charles André, F-69561 St. Genis Laval Cedex, France
}

Received January 5; accepted February 2, 1999

\begin{abstract}
New close sub-systems are discovered in 8 visual late-type double stars. Elements of spectroscopic orbits (4 double-lined and 4 single-lined) are given for the components of quadruple systems ADS 1134 and 11163, for the components of triple systems $\operatorname{ADS} 455,497,5436$, 8236,10044 , and for the primary of the optical pair ADS 9444. Magnitudes, colors, spectral types, masses of individual components are estimated by combining all available data with Hipparcos parallaxes. Among these stellar systems we note a subdwarf triple ADS 497, an old system with subgiant components ADS 10044, and a visual triple ADS 11163 with additional very low mass (possibly substellar) spectroscopic companion in a 4.6 day orbit. The astrometric orbit of ADS $8236 \mathrm{~B}$ with a semimajor axis of 30 mas and a period of $4.6 \mathrm{yr}$ may be recoverable from Hipparcos data.
\end{abstract}

Key words: stars: binaries: spectroscopic - stars: binaries: visual

\section{Introduction}

This work continues the publications of spectroscopic orbits for the components of late-type multiple stars observed with CORAVEL-type radial velocity spectrometer. In our first paper (Tokovinin 1997a - hereafter Paper I) the motivation for this observing program and the methods of data analysis are described. The next 6 orbits were published in (Tokovinin 1998a). Here we present another 8 orbits, following the same strategy which need not be repeated. The results of this study will be incorporated in the current version of Multiple Star Catalogue (Tokovinin 1997b).

\footnotetext{
* Table 4 is only available in electronic form at the CDS via anonymous ftp to cdsarc.u-strasbg.fr (130.79.128.5) or via http://cdsweb.u-strasbg.fr/Abstract.html
}

Table 1. Object identification

\begin{tabular}{lllll}
\hline \hline IDS(1900) & ADS & HD/BD & HIP & Other \\
\hline $00274+2744$ & 455 & 2942 & 2583 & HR 134 \\
$00307+2927$ & 497 & 3266 & 2844 & LTT 10205 \\
$01200+3102$ & 1134 & $8610 / 24$ & $6668 / 75$ & STT 30 \\
$06399+5549$ & 5436 & $48766 / 67$ & 32609 & HR 2486/85 \\
$11311+5642$ & 8236 & 100831 & 56622 & STF 1553 \\
$14520+2953$ & 9444 & 132049 & - & STF 1893 \\
$16206+3716$ & 10044 & 148086 & 80349 & STF 2044 \\
$18199+8434$ & 11163 & +84409 & - & HU 65 \\
\hline \hline
\end{tabular}

A correlation Radial Velocity Meter (RVM) (Tokovinin 1987) was used for the measurements. Most of observations which form the basis of this work were made in 1995-1998 with the 70-cm telescope located on the Moscow University campus and with the 1-m telescope of the Simeis Observatory in Crimea. Velocity zero point was determined by observations of several IAU velocity standards each night. The precision of our velocities ranges from $0.3 \mathrm{~km} \mathrm{~s}^{-1}$ for bright stars to $1.4 \mathrm{~km} \mathrm{~s}^{-1}$ for faint objects or for low-contrast dips. Some observations were also made by the author in 1994 with the CORAVEL spectrometer (Baranne et al. 1979) at the Haute Provence Observatory.

\section{Basic data}

Table 1 contains the identification data on the 8 systems: IDS (1900) index, ADS number (Aitken 1932), HD or BD number, Hipparcos number (ESA 1997), and other identifiers, e.g. double star discoverer codes. The ADS number happens to be a common identifier for all these systems and it will be used throughout this paper.

Basic data on system components (spectral types, visual magnitudes and $B-V$ colors) are given in the left columns of Table 2 and were collected from the literature or from SIMBAD. Most of the photometry is taken from Tycho catalogue (ESA 1997). Proper motions and trigonometric parallaxes are taken from Hipparcos catalogue for 
all but 2 systems. The last 4 columns of Table 2 summarize the results of our study and contain the mean radial velocities, the number of measurements, the mean equivalent widths $E W$ of the cross-correlation (CC) dip, and the projected axial rotational velocities $V \sin i$, as found from the width of CC dip. The method of $V \sin i$ determination and the dependence of $E W$ on $B-V$ color and metallicity are described in Paper I and in (Tokovinin 1990). Parameters of CC dip and the number of measurements are given separately for both components of the doublelined systems.

\section{Spectroscopic orbits and system parameters}

The final orbital elements were found by least-squares fitting with weights inversely proportional to the square of formal velocity errors. The elements and their formal errors are given in Table 3. For circular orbits I checked that non-zero eccentricity did not result in the significant improvement of the quality of fit, and give in Table 3 the upper limits of eccentricity (95\% confidence) as defined by Lucy \& Sweeny (1971). Number of measurements used (primary and secondary lines counted separately) and the rms error of unit weight are given in the 9 th column of Table 3. Its last column contains mass function for singlelined systems or $M \sin ^{3} i$ for the components of doublelined systems. Radial velocity curves are given in Fig. 1. Individual observations, their errors and residuals can be found in Table 4 which is available in electronic form only. It contains also the measurements of non-variable components (a total of 382 velocities of 20 components). The 7 CORAVEL measurements are marked as COR in the last column. The 12 measurements rejected in orbit computations are marked by colons.

For the components with apparently constant velocities, the detection threshold on amplitude is from 0.5 to $2 \mathrm{~km} \mathrm{~s}^{-1}$ (50\% chance of detection). The detection model developed in Tokovinin (1992) indicates that the threshold is about $5 \sigma$, where $\sigma$ is the error of the mean velocity as given in Table 2 .

Magnitude difference of the components of doublelined systems was determined by fitting simultaneously the ratio of dip equivalent widths $E W$ and the combined $B-V$ color, as in Paper I.

The models of multiple systems are given in Table 5 . The first column contains ADS numbers, parallaxes and distance moduli. The second column gives component identification (visual components are marked by uppercase letters, spectroscopic components by second lowercase letters). Then in Cols. 3 to 6 the estimated visual magnitudes, spectral types, and masses of components are given. These estimates come from the magnitude differences of double-lined systems and from spectral type assignments that would match absolute magnitude, observed colors and equivalent widths. Thus the data in the left half of Table 5 represent an "educated guess" based on the available observational data, not the directly measured quantities. Lower limits of secondary mass are given for single-lined systems.

Each hierarchical multiple system can be decomposed into a number of binary systems. The last four columns of Table 5 summarize the parameters of these "elementary" binaries. Column "type" has obvious coding: CPM for common proper motion pairs, VB for visual binaries, SB1 and SB2 for single- and double-lined spectroscopic binaries. The periods of CPM pairs are estimated by the third Kepler law from separation and total mass.

\section{Comments on individual systems}

ADS $455=$ HR 134. The bright primary is a K0III giant which was considered as a spectroscopic binary in the Bright Star Catalogue (Hoffleit \& Warren 1991 - BS). In fact its radial velocity is constant within $\pm 0.5 \mathrm{~km} \mathrm{~s}^{-1}$, as shown by our measurements, the old data and the equality with $\gamma$-velocity of Bab. The small relative motion of $\mathrm{AB}$ since its discovery in 1847 and equal radial velocities indicate that this is a physical system.

The measurements of a faint secondary at $8.5^{\prime \prime}$ from A were extremely difficult, being possible only on nights with good seeing. Although the velocity was seen to be variable already in 1994, only now are we able to derive an orbit. The faint secondary B was classified as K0V, which matches its color $B-V=0.83$. However, our orbit predicts the minimum masses of 0.87 and $0.84 M_{\odot}$ implying somewhat earlier spectral types, G6V and G8V. Assuming these spectral types (which match also the dip $E W$ ), we run into another problem, because at the distance given by Hipparcos a pair of such stars must be $0.7^{\mathrm{m}}$ brighter than observed. A possible explanation might invoke interor circumstellar absorption $A_{V}=0.7^{\mathrm{m}}$, which would also account for the redder color. Alternatively, the errors in parallax and photometry may be at the origin of this discrepancy. Eclipses may be searched in this system, because the inclination $i$ is close to $90^{\circ}$. Axial rotation is synchronized with orbit, since the expected equatorial velocity $6.7 \mathrm{~km} \mathrm{~s}^{-1}$ is close to the actually measured $V \sin i$. A 7day dwarf binary is expected to have circular orbit; small but significant eccentricity of ADS 455B can be explained by the perturbations from the visual component A (Mazeh 1990).

ADS 497. We discovered the close sub-system independently, realizing later that an identical single-lined orbit has already been published by Latham et al. (1988). However, we also measure the secondary dip, and publish here a double-lined orbit which incorporates the data of Latham et al. These two data sets are comparable in quality (rms unit-weight residuals for the primary are 0.53 and $0.65 \mathrm{~km} \mathrm{~s}^{-1}$ for the 23 and 25 observations of RVM and CfA spectrometers, respectively), with no significant 

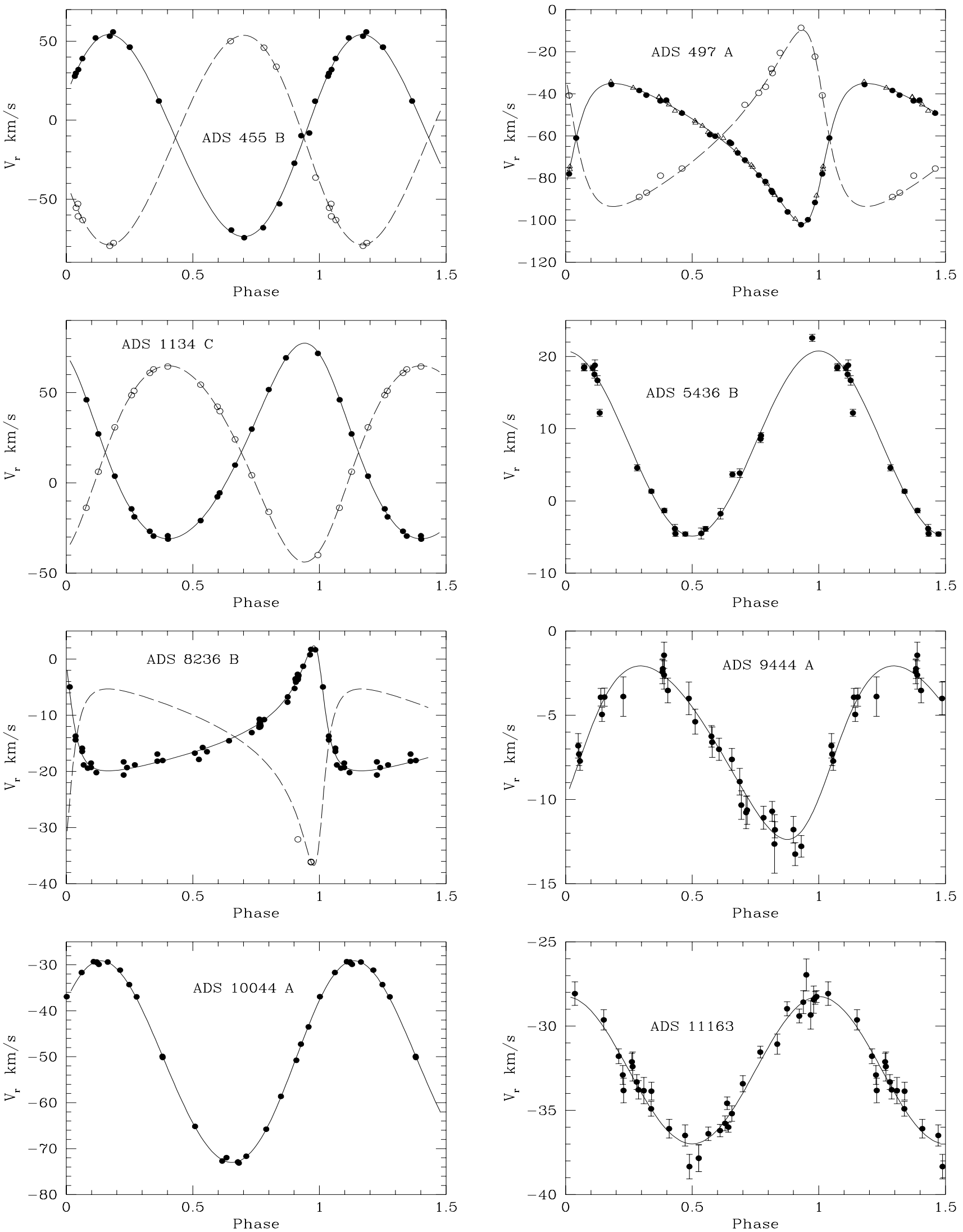

Fig. 1. Radial velocity curves. Radial velocities of primary components are plotted as filled circles and full lines, those of secondary components as empty circles and dashed lines. For ADS 497A the data of Latham et al. (1988) are plotted as triangles. Error bars are shown only for orbits with low amplitudes 
Table 2. Basic observational data

\begin{tabular}{|c|c|c|c|c|c|c|c|c|c|c|c|}
\hline$\overline{\mathrm{ADS}}$ & Comp. & $\begin{array}{l}\text { Sp. } \\
\text { type }\end{array}$ & $\bar{V}$ & $\overline{B-V}$ & $\begin{array}{c}\mu_{\alpha} \\
\operatorname{mas} / y\end{array}$ & $\begin{array}{c}\mu_{\delta} \\
\mathrm{mas} / \mathrm{y}\end{array}$ & $\begin{array}{c}\pi \\
\text { mas }\end{array}$ & $\begin{array}{c}V_{\mathrm{r}} \\
\mathrm{km} \mathrm{s}^{-1}\end{array}$ & $\overline{N_{\text {obs }}}$ & $\begin{array}{c}E W \\
\mathrm{~km} \mathrm{~s}^{-1}\end{array}$ & $\begin{array}{c}V \sin i \\
\mathrm{~km} \mathrm{~s}^{-1}\end{array}$ \\
\hline \multirow[t]{3}{*}{455} & $\mathrm{~A}$ & K0III & 6.30 & 1.00 & \multirow[t]{3}{*}{-4} & \multirow[t]{3}{*}{6} & \multirow[t]{3}{*}{$6.9 \pm 1.0$} & $-11.3 \pm 0.1$ & 9 & $3.87 \pm 0.05$ & $<2$ \\
\hline & B & K0V & 11.26 & 0.83 & & & & $-11.2 \pm 0.1$ & 18 & $1.74 \pm 0.13$ & $6.6 \pm 1.9$ \\
\hline & & & & & & & & & 10 & $1.24 \pm 0.14$ & $<8$ \\
\hline \multirow[t]{3}{*}{497} & $\mathrm{~A}$ & \multirow[t]{3}{*}{$\mathrm{G} 2 \mathrm{~V}$} & 8.41 & 0.66 & 186 & -408 & \multirow[t]{3}{*}{$18.9 \pm 1.7$} & $-60.9 \pm 0.4$ & 23 & $1.58 \pm 0.04$ & 0 \\
\hline & & & & & & & & & 13 & $0.43 \pm 0.05$ & 0 \\
\hline & B & & 9.13 & 0.70 & 181 & -399 & & $-60.0 \pm 0.4$ & 9 & $1.98 \pm 0.09$ & 0 \\
\hline \multirow[t]{3}{*}{1134} & $\mathrm{AB}$ & F9V & 8.07 & 0.63 & 154 & -48 & \multirow{3}{*}{$\begin{array}{l}16.6 \pm 1.5 \\
17.3 \pm 1.0\end{array}$} & $17.2 \pm 0.2$ & 8 & $2.68 \pm 0.06$ & $4.0 \pm 0.7$ \\
\hline & $\mathrm{C}$ & G3V & 8.08 & 0.68 & 155 & -51 & & $16.9 \pm 0.1$ & 17 & $1.33 \pm 0.03$ & 0 \\
\hline & & & & & & & & & 15 & $1.31 \pm 0.03$ & $<4$ \\
\hline \multirow[t]{3}{*}{5436} & $\mathrm{~A}$ & F6V & 6.32 & 0.48 & 58 & -105 & \multirow[t]{3}{*}{$21.3 \pm 1.3$} & $5.7 \pm 0.3$ & 22 & $1.93 \pm 0.03$ & $11.7 \pm 0.2$ \\
\hline & B & F5V & 6.28 & 0.47 & & & & $7.9 \pm 0.2$ & 21 & $2.09 \pm 0.04$ & $17.4 \pm 0.3$ \\
\hline & $\mathrm{C}$ & & 11.2 & & -11 & -7 & & $28.7 \pm 0.8$ & 1 & $1.79 \pm 0.35$ & $<10$ \\
\hline \multirow[t]{3}{*}{8236} & $\mathrm{~A}$ & G5 & 7.72 & 0.66 & -178 & $\begin{array}{l}-92 \\
-9\end{array}$ & \multirow[t]{3}{*}{$22.9 \pm 1.4$} & $-16.0 \pm 0.1$ & 45 & $2.81 \pm 0.03$ & $3.7 \pm 0.4$ \\
\hline & B & & 8.22 & 0.75 & -179 & -109 & & $-13.9 \pm 0.1$ & 49 & $2.82 \pm 0.03$ & $3.9 \pm 0.4$ \\
\hline & & & & & & & & & 3 & $0.38 \pm 0.10$ & \\
\hline \multirow[t]{2}{*}{9444} & $\mathrm{~A}$ & F8 & 8.9 & & -51 & -28 & \multirow[t]{2}{*}{-} & $-6.8 \pm 0.1$ & 29 & $2.22 \pm 0.07$ & $10.9 \pm 0.6$ \\
\hline & B & K4.5 & 10.7 & & & & & $-15.5 \pm 0.2$ & 16 & $4.67 \pm 0.23$ & $5.5 \pm 1.4$ \\
\hline \multirow[t]{2}{*}{10044} & A & K0 & 8.40 & 0.98 & -104 & 12 & \multirow[t]{2}{*}{$9.5 \pm 1.7$} & $-51.5 \pm 0.1$ & 23 & $3.81 \pm 0.04$ & 0 \\
\hline & B & & 8.74 & 0.71 & & & & $-51.8 \pm 0.2$ & 17 & $2.93 \pm 0.06$ & 0 \\
\hline 11163 & $\mathrm{AB}$ & G5 & 9.8 & & -59 & 6 & - & $-32.6 \pm 0.1$ & 34 & $2.08 \pm 0.05$ & $7.0 \pm 0.5$ \\
\hline
\end{tabular}

Table 3. Orbital elements

\begin{tabular}{|c|c|c|c|c|c|c|c|c|c|c|}
\hline \multirow{2}{*}{$\begin{array}{l}\text { ADS } \\
\text { HD } \\
455 B\end{array}$} & \multirow{2}{*}{$\begin{array}{l}P \\
\text { days } \\
7.48866\end{array}$} & \multirow{2}{*}{$\begin{array}{l}T \\
\text { JDH } \\
51060.558\end{array}$} & \multirow{2}{*}{$\begin{array}{l}e \\
0.055\end{array}$} & \multirow{2}{*}{$\begin{array}{l}\omega \\
\circ \\
293.4\end{array}$} & \multirow{2}{*}{$\begin{array}{l}K_{1} \\
\mathrm{~km} \mathrm{~s}^{-1} \\
64.01\end{array}$} & \multirow{2}{*}{$\begin{array}{l}K_{2} \\
\mathrm{~km} \mathrm{~s}^{-1} \\
66.34\end{array}$} & \multirow{2}{*}{$\begin{array}{l}\gamma \\
\mathrm{km} \mathrm{s}^{-1} \\
-11.16\end{array}$} & \multirow{2}{*}{$\begin{array}{l} \\
\sigma_{V} \\
28\end{array}$} & \multicolumn{2}{|c|}{$\begin{array}{c}f(m) \text { or } M \sin ^{3} i \\
M_{\odot} \\
\end{array}$} \\
\hline & & & & & & & & & 0.870 & 0.839 \\
\hline 2942B & \pm 0.00009 & \pm 0.162 & \pm 0.007 & \pm 8.1 & \pm 0.49 & \pm 0.65 & \pm 0.31 & $1.37 / 0.87$ & \pm 0.019 & \pm 0.016 \\
\hline 497A & 35.99878 & 51067.968 & 0.450 & 240.5 & 33.32 & 41.85 & -60.94 & 61 & 0.627 & 0.499 \\
\hline 3266 & \pm 0.00025 & \pm 0.042 & \pm 0.004 & \pm 0.7 & \pm 0.17 & \pm 0.60 & \pm 0.10 & $0.58 / 1.42$ & \pm 0.018 & \pm 0.008 \\
\hline $1134 \mathrm{C}$ & 14.9095 & 51057.552 & 0.132 & 27.6 & 54.17 & 54.40 & 16.90 & 32 & 0.956 & 0.955 \\
\hline 8624 & \pm 0.0109 & \pm 0.061 & \pm 0.004 & \pm 1.4 & \pm 0.29 & \pm 0.34 & \pm 0.13 & $0.67 / 0.53$ & \pm 0.069 & \pm 0.062 \\
\hline 5436B & 4.25856 & 50551.754 & 0 & 0 & 12.82 & & 7.94 & 21 & 0.00093 & \\
\hline 48766 & \pm 0.00024 & \pm 0.016 & $<0.04$ & & \pm 0.24 & & \pm 0.19 & 0.72 & \pm 0.00006 & \\
\hline 8236B & 1690.15 & 49005.39 & 0.648 & 45.1 & 11.13 & 15.72 & -13.85 & 52 & 0.88 & 0.62 \\
\hline 100831B & \pm 4.45 & \pm 3.59 & \pm 0.013 & \pm 1.6 & \pm 0.27 & \pm 1.40 & \pm 0.12 & $0.72 / 0.07$ & \pm 0.17 & \pm 0.07 \\
\hline 9444A & 3237.7 & 48672 & 0.145 & 238 & 5.15 & & -6.82 & 29 & 0.044 & \\
\hline 132049 & \pm 77.0 & \pm 150 & \pm 0.039 & \pm 17 & \pm 0.22 & & \pm 0.14 & 0.65 & \pm 0.005 & \\
\hline $10044 \mathrm{~A}$ & 21.5874 & 50646.729 & 0.034 & 307.4 & 21.95 & & -51.52 & 23 & 0.0236 & \\
\hline 148086 & \pm 0.0032 & \pm 0.642 & \pm 0.007 & \pm 10.7 & \pm 0.12 & & \pm 0.10 & 0.36 & \pm 0.0003 & \\
\hline 11163 & 4.63629 & 50655.681 & 0 & 0 & 4.37 & & -32.62 & 34 & 0.00004 & \\
\hline- & \pm 0.00066 & \pm 0.046 & $<0.08$ & & \pm 0.16 & & \pm 0.11 & 0.61 & - & \\
\hline
\end{tabular}

difference of zero points. This triple system belongs to the Arcturus moving group and may be about $10^{10}$ yrs old (Eggen 1971). Hipparcos astrometry and new radial velocities only confirm this conclusion, giving the galactic velocity $U, V, W=18,-113,-56 \mathrm{~km} \mathrm{~s}^{-1}$. The components are located on the Main Sequence close to the turning point of the oldest clusters, and in few billion years the primary will start to expand. Our analysis of dip contrast gives metallicity $[\mathrm{Fe} / \mathrm{H}]=-0.6$ for both components, whereas Latham et al. (1988) give $[\mathrm{Fe} / \mathrm{H}]=-1.16$.

ADS 1134. This object was placed on the observing program only in 1998, and the double-lined nature of $\mathrm{C}$ was discovered immediately. The velocity amplitudes and dip parameters of $\mathrm{Ca}$ and $\mathrm{Cb}$ are indistinguishable. Minimum masses are close to those estimated from spectral types, and eclipses are hence possible $\left(i \approx 90^{\circ}\right)$, although Hipparcos did not detect any brightness variations. 
Table 5. Models of multiple systems

\begin{tabular}{l|lllll|llll}
\hline \hline $\begin{array}{l}\text { ADS } \\
\pi, \text { mas } \\
m-M\end{array}$ & Comp. & $V$ & $B-V$ & $\begin{array}{l}\text { Sp. } \\
\text { type }\end{array}$ & $\begin{array}{l}\text { Mass, } \\
M_{\odot}\end{array}$ & Sys. & Type & Period & $\begin{array}{l}\text { Sep. } \\
\text { " }\end{array}$ \\
\hline $\mathbf{4 5 5}$ & A & 6.30 & 1.00 & K0III & $2.5:$ & AB & CPM & $18000 \mathrm{y}$ & 8.5 \\
6.9 & $\mathrm{Ba}$ & 11.80 & 0.69 & G6V & 0.88 & Bab & SB2 & $7.5 \mathrm{~d}$ & 0.0006 \\
5.8 & $\mathrm{Bb}$ & 12.27 & 0.74 & G8V & 0.84 & & & & \\
\hline $\mathbf{4 9 7}$ & $\mathrm{Aa}$ & 8.63 & 0.66 & G4V & 0.94 & AB & CPM & $3700 \mathrm{y}$ & 6.2 \\
18.9 & $\mathrm{Ab}$ & 10.23 & 0.87 & K2V & 0.75 & Aab & SB2 & $36.0 \mathrm{~d}$ & 0.005 \\
3.6 & $\mathrm{~B}$ & 9.13 & 0.71 & G7V & 0.85 & & & & \\
\hline $\mathbf{1 1 3 4}$ & $\mathrm{A}$ & 8.11 & 0.60 & F9V & 1.14 & AC & CPM & $105000 \mathrm{y}$ & 56.8 \\
16.6 & $\mathrm{~B}$ & 11.73 & 1.2 & K6V & 0.65 & AB & CPM & $3500 \mathrm{y}$ & 4.5 \\
3.9 & $\mathrm{Ca}$ & 8.83 & 0.68 & G3V & 0.98 & Cab & SB2 & $14.9 \mathrm{~d}$ & 0.003 \\
& $\mathrm{Cb}$ & 8.83 & 0.68 & G3V & 0.98 & & & & \\
\hline $\mathbf{5 4 3 6}$ & $\mathrm{A}$ & 6.32 & 0.48 & F6V & 1.25 & AB & CPM & $2000 \mathrm{y}$ & 4.6 \\
21.3 & $\mathrm{Ba}$ & 6.28 & 0.48 & F6V & 1.25 & Bab & SB1 & $4.25 \mathrm{~d}$ & 0.001 \\
3.4 & $\mathrm{Bb}$ & $17 ?$ & & M7V? & $>0.13$ & & & & \\
\hline $\mathbf{8 2 3 6}$ & $\mathrm{A}$ & 7.72 & 0.66 & G3V & 0.98 & AB & CPM & $2800 \mathrm{y}$ & 6.1 \\
22.9 & $\mathrm{Ba}$ & 8.32 & 0.72 & G7V & 0.88 & Bab & SB2 & $4.6 \mathrm{y}$ & 0.073 \\
3.2 & $\mathrm{Bb}$ & 10.82 & 1.11 & K7V & 0.62 & & & & \\
\hline $\mathbf{1 0 0 4 4}$ & $\mathrm{Aa}$ & 8.40 & 0.98 & K0IV & 1.1 & AB & CPM & $16000 \mathrm{y}$ & 8.3 \\
9.5 & $\mathrm{Ab}$ & $15 ?$ & & M2V? & $>0.37$ & Aab & SB1 & $21.7 \mathrm{~d}$ & 0.002 \\
5.1 & $\mathrm{~B}$ & 8.74 & 0.71 & G6IV & 1.0 & & & & \\
\hline $\mathbf{1 1 1 6 3}$ & $\mathrm{Aa}$ & 9.7 & - & G5V & 0.9 & AC & CPM & $14000 \mathrm{y}$ & 9.2 \\
12 & $\mathrm{Ab}$ & - & - & M8V? & $>0.05$ & AB & VB & $1000 \mathrm{y}$ & 1.5 \\
4.6 & $\mathrm{~B}$ & 10.5 & - & K0V? & 0.8 & Aab & SB1 & $4.6 \mathrm{~d}$ & 0.001 \\
& $\mathrm{C}$ & 12.2 & - & K6V? & 0.6 & & & & \\
\hline \hline
\end{tabular}

Both for $\mathrm{A}$ and $\mathrm{C}$ the $E W$ of dip indicate solar metallicity. The spectral type of the visual secondary B given in Table 5 was assigned to match the magnitude difference. In summary, ADS 1134 presents a quadruple system composed of solar-type dwarf stars with perfectly normal masses and luminosities.

ADS $5436=$ HR 2486/85. The visual components are so close on the sky and so similar in brightness that they were frequently confused. As shown in the Hipparcos catalogue, the western component, designated as $\mathrm{B}$ in $\mathrm{ADS}$, is slightly brighter, and it is $\mathrm{HR} 2486=$ HD 48766. It is this star that is found here to be a singlelined binary. Some of our measurements were influenced by the light from A entering into the slit, which explains the few deviating points in Fig. 1. Undoubtedly, this problem was common to other observers, and, together with frequent misidentifications, could be the reason why the radial velocity of $\mathrm{A}$ was also considered as variable. In fact it is constant, and the difference of $2.2 \mathrm{~km} \mathrm{~s}^{-1}$ with the $\gamma$-velocity of B can be accounted for by the orbital motion in the wide pair AB. Since the discovery of $A B$ in 1830 the separation decreased from $4.8^{\prime \prime}$ to $4.6^{\prime \prime}$ without change in position angle, indicating either a very eccentric or a highly inclined orbit. The component $\mathrm{C}$ is definitely optical (cf. Table 2), as confirmed by our radial velocity measurement. The $E W$ of $\mathrm{A}$ and $\mathrm{B}$ are compatible with solar metallicity. If $\mathrm{Ba}$ rotates synchronously with orbit, its equatorial velocity must be $14.1 \mathrm{~km} \mathrm{~s}^{-1}$, to be compared with the measured $V \sin i=17.4 \mathrm{~km} \mathrm{~s}^{-1}$.

ADS 8236. This pair of nearby dwarfs was placed on our observing list in 1988 on request of Dr. A.A. Kiselev in order to measure accurately the relative radial velocities of the components. The slow variations of the radial velocity of B were discovered in 1990, and now the two periastron passages of this eccentric $4.6 \mathrm{yr}$ orbit are covered by observations. After calculation of the first preliminary orbital solution it was realized that the secondary must be relatively massive. Indeed, in 1997 we obtained 3 observations of its dip, which has only $2 \%$ contrast. This fixes the secondary mass and enables to estimate the orbital inclination, which is close to $90^{\circ}$. This system is similar to ADS 9167, for which a 2.87 yr spectroscopic orbit has been published by Kiyaeva et al. (1998). In both cases the semimajor axis of the spectroscopic subsystems is large, and the corresponding perturbations could be (but have not been) detected from the precise relative astrometry of the wide pairs. For ADS 8236 the semimajor axis of Bab orbit is 73 mas, and the semimajor axis of Ba motion around the center of gravity is 30 mas. It seems likely that by reprocessing the raw Hipparcos data an astrometric orbit of Bab can be extracted. The Hipparcos double star solutions published so far did not consider the model of visual+astrometric triple systems, and this is why no such systems are found in the catalogue. 
ADS 9444. This system, placed on the program by A.A. Kiselev, is in fact optical, as can be inferred from its fast relative motion. Radial velocities only confirm this assertion. The A component turned out to be a spectroscopic binary with a $8.9 \mathrm{yr}$ period. The minimum mass of $\mathrm{Ab}$ is around $0.5 M_{\odot}$. We do not give system model of ADS 9444 in Table 5. Component B has a dip of high contrast and may be a background giant.

ADS 10044. Also from Kiselev's list, this is actually a physical triple star. Hipparcos parallax places both components well above the Main Sequence: A and B are located at the very beginning of the giant branch of the HR diagram of NGC 188, as given in Fig. 2 of Eggen (1971). Comparison with evolutionary tracks (Schaller et al. 1992) indicates a mass of $\mathrm{A}$ around $1.1 M_{\odot}$ and an age between 5 and 10 billion years. The object belongs to the old disk population, with spatial velocity of $U, V, W=-39,-61,+2 \mathrm{~km} \mathrm{~s}^{-1}$. Recently a triple system of similar evolutionary status, HD 158209, was discussed by Griffin (1997). The orbital period of its close pair, $21.825^{\mathrm{d}}$, is strikingly similar to that of ADS $10044 \mathrm{~A}$, $21.726^{\mathrm{d}}$. Both orbits have small eccentricities; it is possible that they have been partially circularized due to the evolutionary expansion of the primary components. Both these triple systems are at a distance of $100 \mathrm{pc}$, but they have distinctly different spatial velocities and hence do not belong to the same moving group.

ADS 11163. The only available information on this visual triple star, which is found to contain a short-period subsystem, comes from WDS (Worley \& Douglass 1984). The relative position of $\mathrm{AC}\left(\rho=9.2^{\prime \prime}\right)$ is fixed during 60 years, which, coupled with the proper motion of $60 \mathrm{mas} / \mathrm{yr}$, indicates that $\mathrm{C}$ is physical component. The same reasoning applies to the closer pair $\mathrm{AB}\left(\rho=1.5^{\prime \prime}\right)$. Taking the spectral type of the primary to be G5V (with such a short orbital period it can not be a giant), we ascribe the spectral types to $\mathrm{B}$ and $\mathrm{C}$ in order to match their magnitudes (cf. Table 5).

The light of A and B was not separated on the slit, but we believe that it is the A component which is variable. Our model predicts that the relative $E W$ of the A and B dips are $64 \%$ and $36 \%$, respectively, if their light is fully mixed. The velocity amplitude of the combined dip is reduced in the same proportion. The actual amplitudes would be 6.7 or $11.9 \mathrm{~km} \mathrm{~s}^{-1}$, depending on whether it is A or B which is variable. These amplitudes are comparable to the dip FWHM of $14.5 \mathrm{~km} \mathrm{~s}^{-1}$, and variations of dip parameters with orbital phase are to be expected. In the plots of the contrast and width of the dip against the orbital phase the variations are barely detectable, being of the order of $10 \%$. So, we conclude that the short-period subsystem belongs to A. Corrected velocity amplitude of $6.7 \mathrm{~km} \mathrm{~s}^{-1}$ corresponds to the minimum secondary mass of $0.05 M_{\odot}$. The inclination is not known, but this system is candidate for a substellar mass object in a short-period orbit; no such objects have been found so far.

\section{Conclusion}

We present the spectroscopic orbits of eight new subsystems in visual binaries. Of these, only one was known previously, and another one belongs to an optical double star. Remaining 6 systems contribute to the general database on multiple stars with known orbits.

Two systems, ADS 5436 and 11163, have companions with very low masses on 4-day orbits. It was already noted (Tokovinin 1998b) that the periods in the 3 to 6 days range are frequent among multiple stars, much more so than in 6 to 12 days range. The same tendency seems to emerge for the giant planets discovered recently around solar-type stars. It seems natural to suggest that in both cases the orbital evolution was governed by similar processes which first caused orbits to shrink and then froze them at typical periods around 4 days.

Acknowledgements. The author is grateful to the personnel of the Simeis observatory for the possibility to use the $1 \mathrm{~m}$ telescope and to M. Mayor who made available CORAVEL. Some measurements were secured by my colleagues N. Gorynya, N. Samus, M. Smekhov, and M. Sachkov. Comments of the referee, T. Mazeh, have served to improve the paper. The database SIMBAD operated at CDS, Strasbourg, France, was consulted. This work was partially supported by the grant from the Russian program "Astronomy".

\section{References}

Aitken R.G., 1932, New General Catalogue of Double Stars, Carnegie Inst., No. 417

Baranne A., Mayor M., Poncet J.L., 1979, Vistas Astron. 23, 279

Eggen O.J., 1971, PASP 83, 271

ESA, 1997, European Space Agency, SP-1200

Griffin R.F., 1997, Observatory 117, 82

Hoffleit D., Warren W.H., 1991, Preliminary Version of the Bright Star Catalogue. NASA CD-ROM

Kiyaeva O.V., Tokovinin A.A., Kalinichenko O.A., 1998, PAZh 24,868

Latham D.W., Mazeh T., Carney B.W., et al., 1988, AJ 96, 567

Lucy L.B., Sweeny M.A., 1971, AJ 76, 544

Mazeh T., 1990, AJ 99, 675

Schaller G., Schaerer D., Meynet G., Maeder A., 1992, A\&AS 96, 269

Tokovinin A.A., 1987, AZh 64, 196 (SvA 31, 98)

Tokovinin A.A., 1990, PAZh 16, 52 (SvAL 16, 24)

Tokovinin A.A., 1992, A\&A 256, 121

Tokovinin A.A., 1997a, A\&AS 121, 71 (Paper I)

Tokovinin A.A., 1997b, A\&AS 124, 75

Tokovinin A.A., 1998a, PAZh 24, 343 (AL 24, 288)

Tokovinin A.A., 1998b, in: Proc. IAU Coll. 170 Precise Stellar Radial Velocities, Hearnshaw J.B., Scarfe C.D. (eds.), ASP Conf. Ser. (in press)

Worley C.E., Douglass G., 1984, The Washington Double Star Catalog. U.S. Naval Obs. 